\title{
VACUUM-ASSISTED CLOSURE IN THE TREATMENT OF STERNAL WOUND INFECTION AFTER CARDIAC SURGERY
}

\author{
Martin Simek ${ }^{\mathrm{a}}$, Petr Nemec ${ }^{\mathrm{a}}$, Bohumil Zalesak ${ }^{\mathrm{b}}$, Martin Kalab ${ }^{\mathrm{a}}$, Roman Hajek ${ }^{\mathrm{a}}$, \\ Lenka Jecminkova ${ }^{\mathrm{a}}$, Milan Kolar ${ }^{\mathrm{c}}$
}
${ }^{a}$ Department of Cardiac Surgery, Teaching Hospital and Palacky University Faculty of Medicine and Dentistry, Olomouc, Czech Republic
${ }^{b}$ Department of Plastic and Aesthetic Surgery, Teaching Hospital and Palacky University Faculty of Medicine and Dentistry, Olomouc, Czech Republic
c Department of Microbiology, Teaching Hospital and Palacky University Faculty of Medicine and Dentistry, Olomouc, Czech Republic
e-mail:martin.simek@c-mail.cz

Received: September 15, 2007; Accepted: October 11, 2007

Key words: Vacuum assisted closure/Sternal wound infection/Cardiac surgery

Aim: Vacuum-assisted closure (VAC) was primarily designed for the treatment of pressure ulcers or chronic, debilitating wounds. Recently, VAC has become an encouraging treatment modality for sternal wound infection after cardiac surgery, providing superior results to conventional treatment strategies.

Methods: From November 2004 to September 2006, 34 patients, undergoing VAC therapy for sternal wound infection following cardiac surgery, were prospectively evaluated. Ten patients $(29 \%)$ were treated for superficial sternal wound infection and 24 (71 \%) for deep sternal wound infection. The median age was 69.9 years (range 48 to 82 ) and the median BMI was $33.4 \mathrm{~kg} / \mathrm{m}^{2}$ (range 28 to 41 ). Twenty patients (59\%) were women and 19 patients (59\%) were diabetics. Owing to sternal wound infection complications, 16 patients (47\%) were readmitted to the department. VAC was used following the previous failure of the conventional treatment strategy in 7 patients $(21 \%)$.

Results: Thirty-three patients (97\%) were treated successfully. One patient (3\%) died of multiple organ failure. The overall length of hospitalization was 34.6 days (range 9 to 62 ). The median number of dressing changes was 4.6 (range 3 to 10). The median VAC treatment time until surgical closure was 9.2 days (range 6 to 21 days). VAC therapy was solely used as a bridge to definite wound closure. Three patients ( $9 \%$ with chronic fistula were re-admitted 1 to 6 months after VAC therapy.

Conclusions: VAC therapy is a safe and reliable option in the treatment of sternal wound infection in cardiac surgery. VAC therapy should be considered an effective adjunct to conventional treatment modalities for the treatment of extensive and life-threatening wound infections following cardiac surgery, particularly in the presence of risk factors.

\section{INTRODUCTION}

Sternal wound infection following cardiac procedures occurs in 1 to $5 \%$ of cases and represents a serious problem involving prolonged hospitalization, increased hospital costs, and increased morbidity and mortality ${ }^{1-4}$. Conventional treatment modalities usually involve surgical debridement with open dressing or closed irrigation or delayed reconstruction ${ }^{5,6}$. The vacuum-assisted closure (VAC) system was introduced into clinical practice for the treatment of pressure ulcers and chronic debilitating wounds in $1997^{7}$. Recent studies have reported the effectiveness of the VAC system in the treatment of sternal wound infection in cardiac surgery, with emphasis on the treatment of deep sternal wound infection ${ }^{8-11}$.

\section{Patients and methods}

From November 2004 to September 2006, 34 patients, undergoing VAC therapy for sternal wound infection following cardiac surgery, were prospectively evaluated. Data obtained from medical records included demographic characteristics are presented in Table 1. The type of cardiac procedure, time interval of wound infection presentation after the procedure, time of VAC therapy, the number of dressing changes and length of hospitalization are summarized in Table 2. Ten patients (29\%) were treated for superficial sternal wound infection (SSWI) and twentyfour (71\%) for deep sternal wound infection (DSWI).

\section{Sternal wound infection classification and predictors of increased risk factor for sternal wound infection}

Sternal wound infection was defined according to the guidelines of the Centre for Disease Control and Prevention $(\mathrm{CDC})^{12}$. Involvement of skin or subticular tissue was defined as a SSWI, involvement of deeper tissue such as a pectoral fascia, sternal bone as well as mediastinal space was classified as a DSWI. Diagnosis of sternal wound infection required at least one of the following criteria: (1) an organism was isolated from the culture or mediastinal fluid; (2) evidence of wound infection during the 


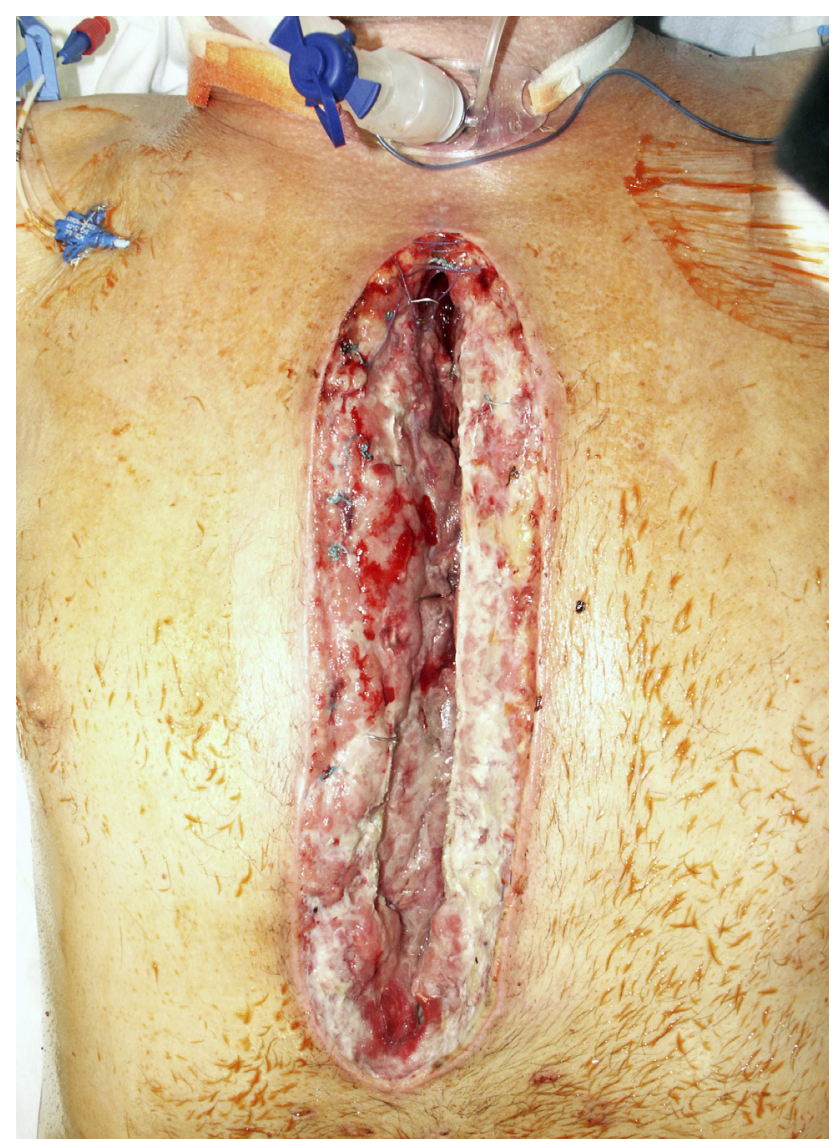

Fig. 1. Deep sternal wound infection (type II)

operation and (3) one of the following conditions: chest pain, sternal instability, or fever $\left(>38^{\circ} \mathrm{C}\right)$ accompanied by either purulent discharge from the mediastinum or an organism isolated from the blood culture or culture of drainage from the mediastinal space ${ }^{11}$. The simplification of the traditional Oakley-Wright classification of DSWI based on the time of presentation and the presence of risk factors was adopted by the authors ${ }^{13}$. A DSWI occurring within 2 weeks after the operation was classified as type I and a DSWI present 2 to 6 weeks after the operation as type II. Finally, a DSWI present for the first time more than 6 weeks after the procedure was classified as type III. Detailed wound classification types are shown in Table 3. Predictors of increased risk of DSWI included: (1) obesity, (2) pedicled bilateral internal mammary artery grafts in diabetics, prolonged operative time and (4) need for repeated blood transfusion in the early post-operative pe$\operatorname{riod}^{3,4}$.

Extensive and/or massively discharging SSWI requiring frequent dressing changes was primarily managed by the VAC system. In the treatment of the DSWI, the VAC system was initially employed as a treatment modality for failed conventional treatments involving one-step wound debridement, sternal rewiring and application of closed drainage. Later, when the technique was well mastered, the VAC system was routinely used as a first-line treatment for DSWI as well. The identical therapeutic algorithm was adopted for all sternal wound infections. Necrotic tissue

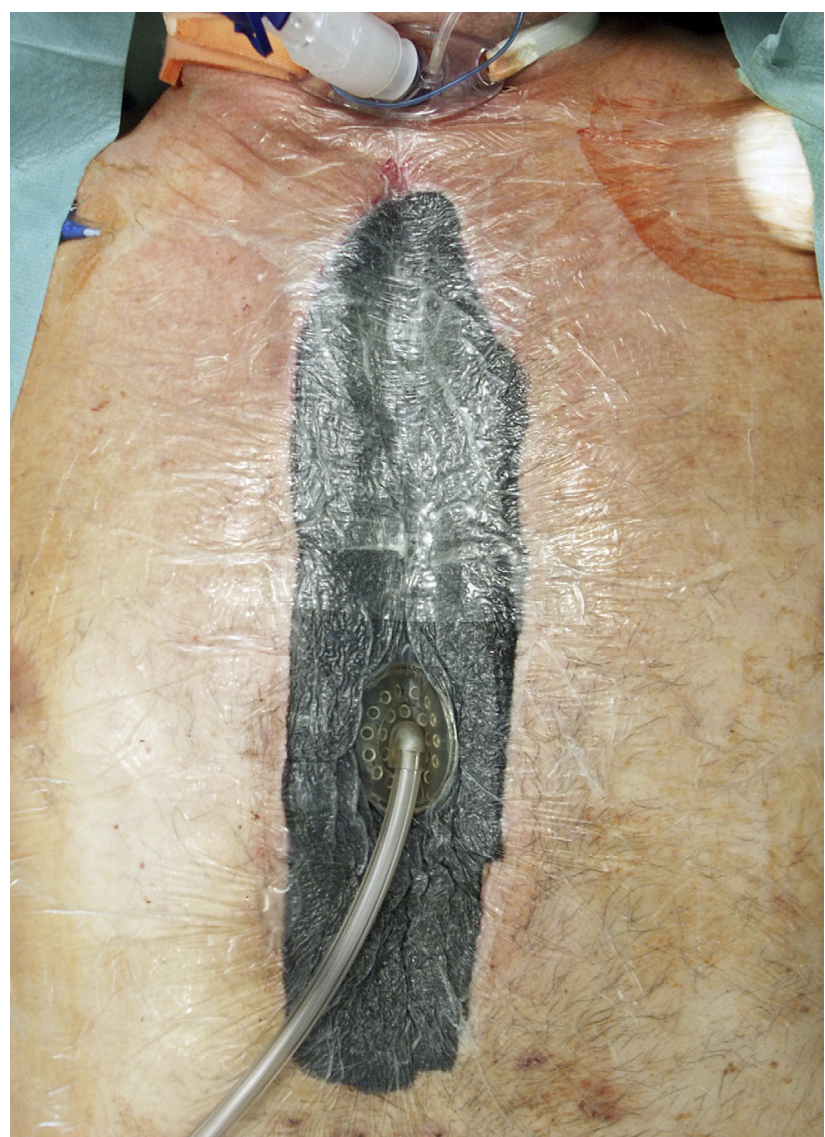

Fig. 2. The VAC system applied into the wound.

and foreign materials were debrided under aseptic conditions and the wound bed was massively irrigated with normal saline solution (Figure 1). Sterile polyurethane foam dressing, with a defined pore structure of 400 to $600 \mu \mathrm{m}$ (Granufoam $^{\circledR}$, KCI, Vienna, Austria), was trimmed to fit between the wound edges. In the case of DSWI, a layer of non-adhesive dressing was applied to the anterior aspect of the heart to protect the free wall of the right ventricle and/or conduits. Subsequently, foam was placed in two layers, on the one placed under the sternal edges the second was superimposed to fill up the whole wound cavity. Tubing was applied to the foam dressing (TRACpad ${ }^{\circledR}$, KCI, Vienna, Austria) and the wound was sealed with a transparent adhesive drape (Figure 2). The drainage tube was connected to a purpose-built vacuum source (VAC ATS ${ }^{\circledR}$ pump unit; KCI, Vienna, Austria). The VAC device is able to generate continuous or intermittent negative pressure variations from 50 to $250 \mathrm{~mm} \mathrm{Hg}$. The target subatmospheric pressure is monitored and maintained at the wound site, even during the patient's movement and breathing. Exudates from the wound were collected into a canister embedded into the pump unit. Initially, $50 \mathrm{~mm}$ $\mathrm{Hg}$ of negative pressure was applied to the adjunct of the foam to the wound geometry. The therapy was subsequently targeted to $125 \mathrm{~mm} \mathrm{Hg}$ continuous negative pressure. The polyurethane foam dressing was changed every 48 to 72 hours. The wound was repeatedly, gently debrided, irrigated with a saline solution and covered in 


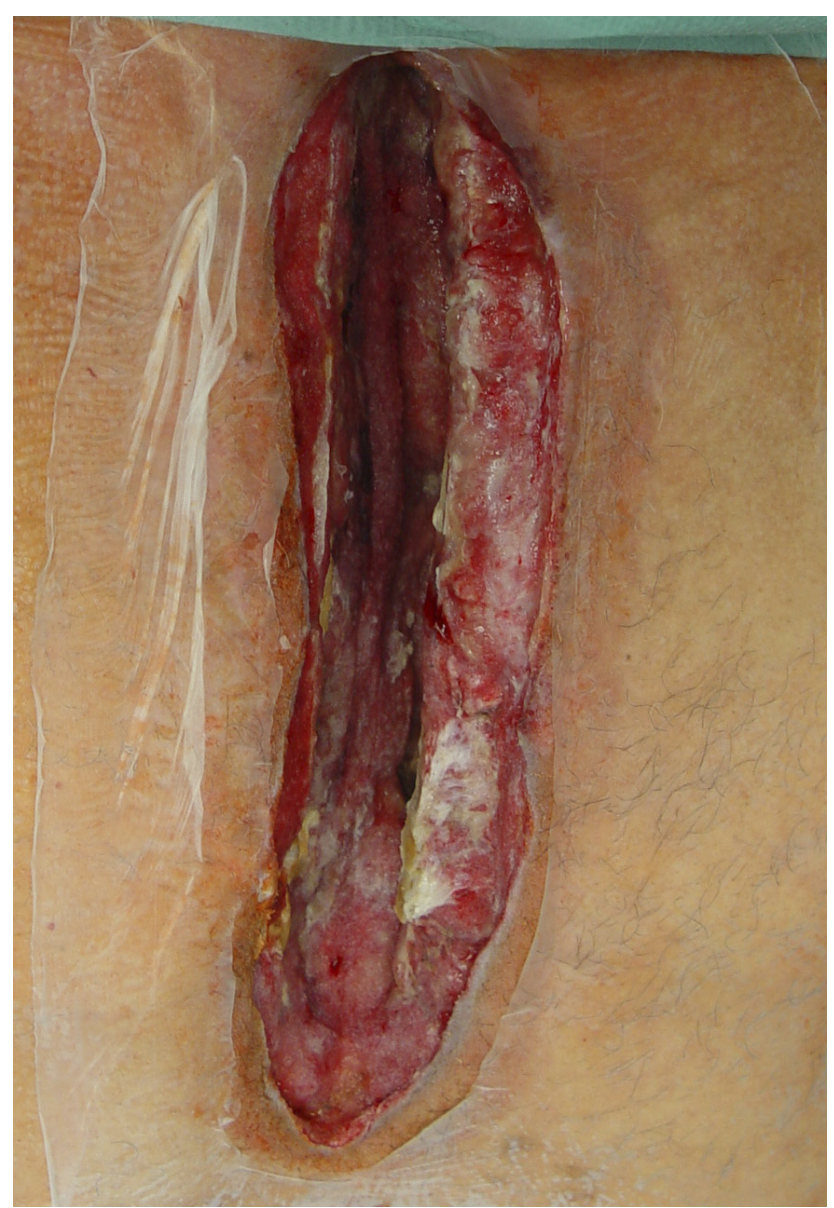

Fig. 3. The result of the VAC therapy. Prepared wound bed prior to the reconstruction.

the same fashion. At least two bacteriological culture samples from the wound were obtained during each dressing change. In case of the DSWI, the wound bed preparation and foam placement were carried out under a short period of general anaesthesia. The patients were extubated immediately after the procedure, if their general condition allowed it. Meticulous nutritional support as well as tight intravenous insulin management was ensured. The antibiotic treatment was given according to the sensitivity of the bacteriological strains and the clinical response of the patient. All patients received a prolonged course of culture-dependent intravenous antibiotics during the VAC treatment. Antibiotics were discontinued when the healing process and parameters of inflammation had diminished except from DSWI when oral antibiotics were continued for 4 weeks after the wound closure. Wound culture characteristics are summarized in Table 4 . The wound was considered to be free of any infection according to advanced local signs of the healing process including the formation of well-vascularised granulation tissue together with negative bacteriological cultures (Figure 3 ). Further, the decline in serological inflammatory parameters, particularly the C-reactive protein level less than $50 \mathrm{mg} / \mathrm{l}$ was required for termination of the VAC therapy. Since excessive residual defects remained, all patients were subsequently indicated for the reconstructive procedure.

\section{RESULTS}

Complete healing was achieved in 33 patients (97\%). One patient suffering from DSWI died (3\%) of multiple organ failure on the $24^{\text {th }}$ postoperative day, despite achieving negative bacteriological cultures during the therapy. The hospital stay ranged from 9 to 62 days (median 34.6 days). Median of the SSI presentation after cardiac procedure was 16 days (range 4 to 52 day). Sixteen (47\%) patients were re-admitted to our department due to sternal infection despite uneventful wound healing at the time of discharge. The median VAC treatment time until surgical closure was 9.2 days (range 6 to 21 days). The wound defects after VAC therapy were managed either by direct wound closure or by local fasciocutaneous and/or myocutaneous advancement flaps based on the size of the residual defect to ensure tension-free closure. In the SSWI group, one partial superficial wound dehiscence after the direct closure was recorded. In addition, one patient was re-admitted for sinus tract formation 6 months after the VAC therapy. Both patients were successfully healed by debridement and major pectoral myocutaneous flap transfer. In the DSWI group, seven patients (29\%) were switched to VAC therapy after failure of the conventional treatment strategy. Afterwards, based on increasing experience, 17 patients with DSWI (71\%) were scheduled for the VAC system application as a first-line procedure. In an effort to preserve chest stability, the subsequent reconstructive procedure in this group involved either complete or partial sternum rewiring, depending on the sternal mass loss during debridement. Simultaneously, any residual soft tissue defect was covered by bilateral major pectoral myocutaneous advancement flap in all cases of DSWI. One partial wound dehiscence was noted in the distal part of sternotomy and managed by a delayed direct wound closure. In addition, 2 patients ( $6 \%$ ) were re-admitted 1 to 6 months after wound closure due to wire-associated chronic sinus tract formation. One or more independent risk factors for the sternal wound infection development were presented in all patients (Table 1). Staphylococcus aureus and coagulase-negative staphylococcus dominated in the spectrum of organisms cultivated from wounds (Table 3), but, no methicilline-resistant staphylococcal strains were cultivated. There was no significant difference in outcome based on etiological causative agent. The continuous VAC therapy reaching subatmospheric pressure up to $125 \mathrm{~mm}$ $\mathrm{Hg}$ caused no patient discomfort. No VAC device-related complications were noted.

\section{DISCUSSION}

The VAC system was initially designed to treat chronic or debilitating wounds ${ }^{7}$. Since the first use of VAC therapy in 1989, this treatment modality has rapidly evolved into a widely accepted treatment for chronic and acute wounds, contaminated wounds, burns, and wound complications from unsuccessful operations ${ }^{8}$. The system combines the benefits of both closed and open wound 
Table 1. Preoperative characteristics $(n=34)$

\begin{tabular}{|l|c|c|}
\hline Variable & value & $\%$ \\
\hline Age $(\mathrm{y})$ & $69.9(48-82)$ & \\
\hline BMI (kg/m2) & $33.4(28-41)$ & \\
\hline M/F ration & $15 / 17$ & $44 / 56$ \\
\hline Diabetes mellitus & 20 & 59 \\
\hline COPD & 6 & 18 \\
\hline CHRF & 6 & 18 \\
\hline Immunosuppressive therapy & 3 & 9 \\
\hline
\end{tabular}

BMI - body mass index

$\mathrm{M} / \mathrm{F}$ - male/female

COPD - chronic obstructive pulmonary disease

$\mathrm{CHRF}$ - chronic renal failure

Table 2. Cardiac procedures and wound care characteristics $(n=34)$

\begin{tabular}{|l|c|c|}
\hline Variable & value & $\%$ \\
\hline CABG & 25 & 74 \\
\hline CABG+AE/MAZE & 5 & 14 \\
\hline CABG (BIMA) & 2 & 6 \\
\hline Valve replacement & $16(3-52)$ & 6 \\
\hline $\begin{array}{l}\text { Time to presentation } \\
\text { of infection (d) }\end{array}$ & $4.6(3-10)$ & \\
\hline $\begin{array}{l}\text { No. of wound dressings } \\
\text { Length of VAC therapy (d) }\end{array}$ & $9.2(6-20)$ & \\
\hline $\begin{array}{l}\text { Overall length } \\
\text { of hospitalization (d) }\end{array}$ & $34.6(9-62)$ & \\
\hline
\end{tabular}

CABG - coronary artery bypass grafting

AE - aneurysmectomy

MAZE - atrial fibrillation treatment

BIMA - bilateral internal mammary artery harvest

treatment. On one hand, the controlled application of subatmospheric pressure to the wound approximates the wound edges and provides a mass filling effect. On the other hand, it has a positive effect in several wound healing processes. It produces arteriolar dilatation, improves microcirculatory flow, reduces wound oedema and bacterial colonization ${ }^{7,8,14,15}$. Generally, it leads to significant acceleration of granulation tissue formation ${ }^{7,8,15}$.

Sternal wound infections remain some of the most feared complications in cardiac surgery. Growing experience with VAC treatment in the field of cardiac surgery is showing its over the conventional treatment of sternal infections including either one-step surgical debridement, sternal rewiring and closed mediastinal drainage or repeated debridement, open packing and subsequent sternal reconstruction ${ }^{8-11,16,17}$. In the VAC group, freedom from mediastinal microbiological cultures was achieved earlier, C-reactive protein level declined more rapidly, in-hospital
Table 3. Wound sternal infection classification $(n=34)$

\begin{tabular}{|l|c|c|}
\hline \multicolumn{1}{|c|}{ Variable } & $\mathrm{n}$ & $\%$ \\
\hline SSWI & 10 & 29 \\
\hline DSWI & 24 & 71 \\
\hline Type I & 9 & 37 \\
\hline Type II & 10 & 43 \\
\hline Type III & 5 & 20 \\
\hline
\end{tabular}

SSWI - superficial sternal wound infection

DSWI - deep sternal wound infection

Table 4. Wound culture characteristics $(n=34)$

\begin{tabular}{|l|c|c|}
\hline Variable & $\mathrm{n}$ & $\%$ \\
\hline Staphylococcus aureus & 20 & 58 \\
\hline CoNS & 9 & 27 \\
\hline Escherichia coli & 1 & 3 \\
\hline Pseudomonas aeruginosa & 3 & 9 \\
\hline Klebsiela oxytoca & 1 & 3 \\
\hline MRSA & 0 & 0 \\
\hline
\end{tabular}

CoNS - coagulase negative staphylococcus

MRSA - methiciline-resistant staphylococcus

stay was shorter, sternal closure was achieved earlier and long-term survival tended to be higher ${ }^{17-20}$. VAC therapy markedly reduced required surgical intervention and reoperations for persistent infections, even in the group of high-risk patients ${ }^{19}$. Beyond the positive effect of the VAC on the local wound healing process, the foam becomes firm through application of subatmospheric pressure and acts as a splint for the sternum ${ }^{10,11}$. This enables mobilization of the patients and allows them either to breathe spontaneously or to be weaned from artificial ventilation during the inherent therapy ${ }^{8,11}$. Further, the stabilisation of the remaining sternal edges may be helpful in preventing right ventricular or conduit injury ${ }^{8-10}$. VAC therapy was primarily used as a bridge to allow cleaning and preparation of the wound bed for further reconstruction $^{8,10,11}$. Use of the VAC therapy as a definitive closure strategy has been recently reported ${ }^{8,21}$. This approach seems to be mostly beneficial in high-risk patients, where it reduces dependence on regional flap and incidence of flap-related morbidity, or in cases of SSWI. Nevertheless, this approach is associated with time extension for the VAC treatment as well as increased cost ${ }^{21}$. Traditionally, timing of the following surgical closure is based on the appearance of the wound, negativity of wound cultures and general patient condition. However, the VAC therapy, 
guided by $\mathrm{C}$-reactive protein level, facilitates timing of wound closure, decreased morbidity and increased costeffectiveness of the procedure ${ }^{22}$. Decline in C-reactive protein levels below $50 \mathrm{mg} / \mathrm{l}$ can be considered a safe trigger for wound closure timing within the institutional algorithm ${ }^{23}$.

The authors present their first experience with VAC treatment for sternal wound infections following cardiac surgery. Despite the limited patient cohort, inhomogeneous group of patients and nonrandomized design, VAC therapy was effective and suitable, particularly in the presence of risk factors such as obesity, diabetes, bilateral internal mammary artery grafting and immunosuppressive therapy. The positive effect of subatmospheric pressure on the local wound healing process, active wound drainage and approximation of the wound edges was advantageous. In the case of DSWI, the VAC system allow us to perform wound debridement repeatedly under brief general anaesthesia and subsequently enabled, due to the stabilizing effect on the sternal halves, spontaneous ventilation and early mobilization of patients. VAC therapy was used as a bridge to definitive surgical closure in all cases. Regarding the extent of tissue loss, the tension-free closure technique was widely accepted with the use of local advancement flaps to cover residual soft tissue defects if needed ${ }^{24}$. However, the sternum-sparing approach, including sternum rewiring for preservation of chest stability, may be associated with later risk of wire-related, chronic sinus tract formation ${ }^{25}$.

\section{CONCLUSION}

The VAC system is a safe and reliable option in the treatment of extensive wound sternal infection in cardiac surgery, mainly promising as the first-line treatment for DSWI, with excellent survival and low failure rate.

\section{REFERENCES}

1. Finkelstein R, Rabino G, Mashiah T, et al. Surgical site infection rates following cardiac surgery: The impact of a 6-year infection control program. Am J Infect Control 2005; 33:450-4.

2. Lepelletier D, Perron S, Bizouarn P, et al Surgical-site infection after cardiac surgery: Incidence, microbiology, and risk factors. Infect Control Hosp Epidemiol 2005; 26:466-72.

3. Ridderstolpe L, Gill H, Granfeldt H, Ahlfeldt H, Rutberg H. Superficial and deep sternal wound complications: incidence, risk factor and mortality. Eur J Cardiothorac Surg 2001; 20:1168-75.

4. Francel TJ, Kouchoukos NT. A rational approach to wound difficulties after sternotomy: The problem. Ann Thorac Surg 2001; 72:1411-8.

5. Ascherman JA, Patel SM, Malhotra SM, Smith CR. Management of sternal wounds with bilateral pectoralis major myocutaneous advancement flaps in 114 consecutively treated patients: Refinements in technique and outcomes analysis. Plast Reconstr Surg 2004; 114:676-83.

6. Kirsch M, Mekontso-Dessap A, Houel R, Giroud E, Hillion ML, Loisance DY. Closed drainage using redon catheters for poststernotomy mediastinitis: Results and risk factors for adverse outcome Ann Thorac Surg 2001; 71:1580-6.
7. Argenta LC, Morykwas MJ. Vacuum-assisted closure. A new method for wound control and treatment: Clinical experience. Ann Plast Surg 1997; 38:563-77.

8. Argenta LC, Morykvas MJ, Marks MW, et al. Vacuum-assisted closure: State of art. Plast Reconstruct Surg 2006; 117: 127-42S

9. Fuchs U, Zittermann A, Stuetten B, Groening A, Minami K, Koefer R. Clinical outcome of patients with deep sternal infection managed by vacuum-assisted closure compared to conventional therapy with open packing: A retrospective analysis. Ann Thorac Surg 2005; 79:526-31.

10. Fleck TM, Fleck M, Moidl R, et al. The vacuum-assisted closure system for the treatment of deep sternal wound infection after cardiac surgery. Ann Thorac Surg 2002; 74:1596-600.

11. Sjoegren J, Gustafsson R, Nilsson J, Malmsjoe M, Ingemansson R. Clinical outcome after poststernotomy mediastinitis: Vacuumassisted closure versus conventional treatment. Ann Thorac Surg 2005; 79:2049-55.

12. Mangram AJ, Horan TC, Pearson ML, Silver LC, Jarvis WR. The hospital infection control practise advisory committee. Guidelines for prevention of surgical site infection. Infect Control Hosp Epidemiol 2002; 20:247-78.

13. El Oakley R, Wright J. Postoperative mediastinitis: Classification and management. Ann Thorac Surg 1996; 61:1030-6

14. Wackenfors A, Sjoegren J, Algotsson L, Gustafsson R, Ingemansson $\mathrm{R}$, Malmsjo M. The effect of vacuum-assisted closure therapy on the pig femoral artery vasomotor responses. Wound Repair Regen 2004; 12:244-251.

15. Wackenfors A, Sjoegren J, Gustafsson R, Algotsson L, Ingemansson R, Malmsjo M. Effects of vacuum-assisted closure therapy on inguinal wound edge microvascular blood flow. Wound Repair Regen 2004; 12:600-606

16. Immer FF, Durrer M, Muehlemann KS, Erni D, Galh B, Carrel TP. Deep sternal wound infection after cardiac surgery: Modality of treatment and outcome. Ann Thorac Surg 205; 80:957-61

17. Doss M, Martens S, Wood JP, Wolff JD, Baier Ch, Moritz A. Vacuum-assisted suction drainage versus conventional treatment in the management of poststernotomy osteomyelitis. Eur $\mathbf{J}$ Cardiothorac Surg 2002; 22:934-38.

18. Sjoegren J, Gustafsson R, Nilsson J, Malmsjoe M, Ingemansson R. The impact of vacuum-assisted closure on long term survival after post-sternotomy mediastinitis. Ann Thorac Surg 2005; 80:12705.

19. Cowan KN, Teague L, Sue SC, Mahoney JL. Vacuum-assisted wound closure of deep sternal infection in high-risk patients after cardiac surgery. Ann Thorac Surg 2005; 80:2205-12.

20. Scholl L, Chang E, Reitz B, Chang J. Sternal osteomyelitis: Use of vacuum-assisted closure device as an adjunct to definitive closure with sternectomy and muscle flap reconstruction. J Card Surg 2004; 19:453-61.

21. Gustafsson RI, Sjoegren J, Ingemansson. Deep sternal wound infection: a sternal-sparing technique with vacuum-assisted closure therapy. Ann Thorac Surg 2003; 76:2048-53.

22. Gustafsson RI, Johnsson P, Algotsson L, Blomquist S, Ingemansson $\mathrm{R}$. Vacuum-assisted closure therapy guided by $\mathrm{C}$-reactive protein level in patients with deep sternal wound infection. J Thorac Cardiovasc Surg 2002; 123:895-900.

23. Sjoegren J, Malmsjoe M, Gustafsson R, Ingemansson R. Poststernotomy mediastinitis: a review of conventional surgical treatments, vacuum-assisted closure therapy and presentation of the Lund University Hospital mediastinitis algorithm. Eur $\mathbf{J}$ Cardiothoracic Surg 206; 30: 898-905.

24. Kletensky J, Padera J. Defects of sternal area. Acta chir plast 2006; 48:5-9.

25. Simek K, Nemec P, Zalesak B, et al. Negative pressure therapy as a treatment modality for surgical site infection in cardiac surgery. Acta chir belg 2007; 107: accepted in press 